
vaginalis

\author{
Araújo, R. D.; Caridade, T. N. S.; Araújo, R. M.* \\ Rev. Virtual Quim., 2018, 10 (5), 1446-1454. Data de publicação na Web: 10 de setembro de 2018 \\ http://rvq.sbq.org.br
}

\title{
Sulfated Polysaccharide from the Marine Sponge Callyspongia vaginalis
}

Abstract: The literature reports several results of chemical investigations from marine ecosystems, which have showed molecules with great biological potential, therefore, its fauna and flora has been target of several studies. Among the marine organisms highlight the sponges, sessile animals, belonging to the poriferous phylum, a profitable source of micro and macromolecules such as polysaccharides, with several biological activities reported in the literature. The chemical investigation of Callyspongia vaginalis led to the isolation of a sulfated polysaccharide. Experiments of hydrolysis, reducing sugars, thermal stability, infrared, and ${ }^{1} \mathrm{H}$ NMR analyses were performed to characterize the polysaccharide. The ideal time for hydrolysis was 6 hours, presenting a concentration of $4.60 \mathrm{mg} / \mathrm{mL}$ of reducing sugar. The polysaccharide showed thermal stability up to about $200^{\circ} \mathrm{C}$. This is the first report of polysaccharide in the genus Callyspongia

Keywords: Callyspongia vaginalis; sulfated polysaccharide; polysaccharide analysis.

\section{Resumo}

A literatura apresenta diversos resultados de investigações químicas em ecossistemas marinhos, que têm apresentado moléculas com grande potencial biológico, por isso, sua fauna e flora tem sido alvo de diversos estudos. Dentre os organismos marinhos destacam-se as esponjas, animais sésseis, antigos, pertencentes ao filo porífera, fonte profícua de micromoléculas e macromoléculas como os polissacarídeos, com diversas atividades biológicas relatadas na literatura. A investigação química de Callyspongia vaginalis levou ao isolamento de um polissacarídeo sulfatado. Experimentos de hidrólise, de açúcares redutores, estabilidade térmica, infravermelho e RMN de ${ }^{1} \mathrm{H}$ foram realizadas para caracterização do polissacarídeo. $O$ tempo ideal para hidrólise foi de 6 horas, apresentando uma concentração de $4,60 \mathrm{mg} / \mathrm{mL}$ de açúcar redutor. O polissacarídeo apresentou estabilidade térmica até cerca de $200^{\circ} \mathrm{C}$. Este é o primeiro relato de polissacarídeo no gênero Callyspongia.

Palavras-chave: Callyspongia vaginalis; polissacarídeo sulfatado; análise de polissacarídeo.

\footnotetext{
* Universidade Federal do Rio Grande do Norte, Instituto de Química, Laboratório de Isolamento e Síntese de Compostos Orgânicos - LISCO, Lagoa Nova, CEP 59078-970, Natal-RN, Brasil.

Mrenat.onca@gmail.com

DOI: $\underline{10.21577 / 1984-6835.20180098}$
} 


\title{
Polissacarídeo Sulfatado da Esponja Marinha Callyspongia vaginalis
}

\author{
Rusceli D. de Araújo, Taíza N. da S. Caridade, Renata M. Araújo*
}

Universidade Federal do Rio Grande do Norte, Instituto de Química, Laboratório de Isolamento e Síntese de Compostos Orgânicos - LISCO, Lagoa Nova, CEP 59078-970, Natal-RN, Brasil.

* renat.onca@gmail.com

Recebido em 18 de maio de 2018. Aceito para publicação em 15 de agosto de 2018

\section{Introdução}

\section{Metodologia}

2.1. Obtenção do polissacarídeo

2.2. Hidrólise do polissacarídeo

2.3. Infravermelho

2.4. Dosagem de açúcar redutor

2.5. Análise termogravimétrica

2.6. Ressonância magnética nuclear

\section{Resultados e Discussão}

3.1. Obtenção do polissacarídeo

3.2. Hidrólise e dosagem de açúcar redutor do polissacarídeo

3.3. Infravermelho

3.4. Análise termogravimétrica

3.5. Ressonância magnética nuclear

\section{Conclusões}

\section{Introdução}

Organismos marinhos representam uma fonte rica de compostos com potencial biológico promissor, destacando-se as micromoléculas, como ácidos graxos, terpenos, policetídeos e alcaloides, que atuam principalmente, como sistema químico de defesa destes seres e as macromoléculas que apresentam potencial biotecnológico promissor, como proteínas e polissacarídeos.
Dentre os organismos marinhos, destacamos as esponjas, estes animais primitivos pertencentes ao filo porífera, são possivelmente os menos desenvolvidos do reino animal, sendo os representantes multicelulares mais simples viventes. ${ }^{1,2}$

Até 2010 haviam sido identificadas 8365 espécies de esponjas, e deste total, $98 \%$ de habitat marinho ${ }^{1}$. O interesse pelas esponjas se intensificou após estudos com a esponja Cryptotethia crypta e o isolamento dos compostos ARA-U e ARA-T, Uracila 
arabinosídeo e Timina arabinosídeo, respectivamente. Estas moléculas conduziram à síntese de um potente anti-retroviral, o zidovudine, também conhecido como AZT. ${ }^{3,4}$ Desde então, as esponjas têm se tornado alvo de estudos químicos e biológicos, com inúmeras substâncias novas reportadas por ano, apresentando diversas atividades biológicas, como: antibiótica, antitumoral, antiviral, anti-inflamatória e antioxidante..$^{5-7}$

Dentre os gêneros de esponjas, podemos destacar o Callyspongia, o qual possui diversas classes de metabólitos já isolados, e muitos com atividade biológica comprovada. Podemos citar o policetídeo calistatina A, isolado de $C$. truncata com atividade citotóxica; ${ }^{8}$ os poliacetilenos sifonodiol e dihidroxi-sifonodiol isolados de $C$. sp e $C$. truncata, assim como os meroterpenos ilhabreno e isoakaterpina, provenientes de $C$. $s p$ com atividade anticâncer; ${ }^{9,10}$ e peptídeos como a foriospongina $B$, isolado de $C$. bilamellata, que possui atividade antiparasitária. ${ }^{11}$ Além destas diversas outras moléculas comprovam a relevância química e biológica de estudos com espécimes pertencentes ao gênero Callyspongia.

As macromoléculas, como os polissacarídeos, também são identificadas e isoladas em esponjas. Quimicamente os polissacarídeos são polímeros naturais em que seus monômeros são unidades de monossacarídeos. Estas moléculas podem ser obtidas a partir de algas marinhas, como também do camarão, caranguejo e esponjas marinhas. ${ }^{12,13}$ Os polissacarídeos participam de diversos processos biológicos como interação e reconhecimento celular.

Uma característica estrutural em polissacarídeos de origem marinha é a presença de grupamento sulfato, ${ }^{14}$ como as gliconectinas, que possuem a capacidade de se aderir à células, gerando uma outra classe de compostos, semelhante a proteoglicanas. ${ }^{15}$ Eles são relatados em diversas espécies de esponjas marinhas e estão associados a atividades antiviral, anti HIV, antioxidantes, antitumorais, dentre outras. ${ }^{16-18}$ Alguns invertebrados marinhos têm se mostrado promissores na obtenção de polissacarídeos sulfatados, com estruturas únicas como Lgalactanas e L-fucanas. Estas moléculas apresentam atividades anticoagulantes e antitrombóticas devido a sua similaridade com a heparina, que é um mucopolissacarídeo sulfatado, com grande quantidade de cargas elétricas negativas e constitui $o$ ácido macromolecular mais forte existente no organismo. Pode ser distinguida de outros polissacarídeos pela sua extrema acidez, decorrente da grande quantidade de radicais sulfatados na sua molécula, ${ }^{18,19}$ um mero de sua estrutura é apresentado na Figura 1 a seguir.
A

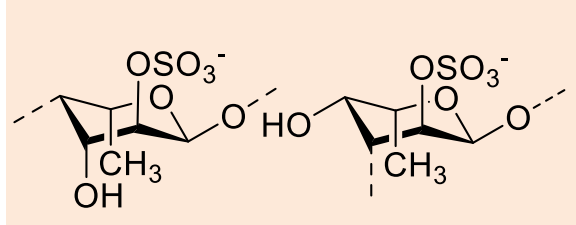

B

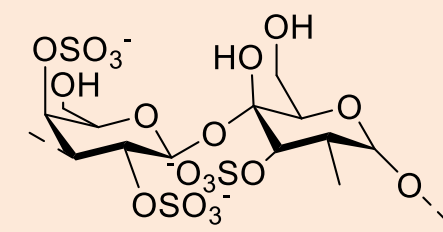

Figura 1: Estruturas de meros de $\alpha$-L-fucanas sulfatadas (A); galactanas (B) sulfatadas.

Tendo em vista o grande potencial biológico que os polissacarídeos apresentam, - presente trabalho apresenta a caracterização parcial do polissacarídeo sulfatado, proveniente da esponja marinha Callyspongia vaginalis. Estudos com este foco não foram relatados anteriormente na literatura para a esponja marinha em questão. Os polissacarídeos foram obtidos de modo simples e seguindo o quinto princípio da química verde, pois utiliza água como solvente extrator. ${ }^{20,21}$ 


\section{Metodologia}

\subsection{Obtenção do polissacarídeo}

Um espécime de Callyspongia vaginalis (2.345 kg de peso molhado) foi coletada no Parque Estadual Marinho da Pedra da Risca do Meio (situado a 10 milhas náuticas do Porto do Mucuripe A, 333,800 'S e 3826,000' W; B, 336.000 'S e 3826.000' W; C, 336.000 'S e 3821.600' W; D, 333.800 'S e 3821.600 'W), a profundidades em torno de $20 \mathrm{~m}$ em 14 de julho de 2004, no estado do Ceará, e identificado pelo prof. Dr. Eduardo Carlos Medina Hajdu, Universidade Federal do Rio de Janeiro Janeiro, sob o número MNRJ 8671 . O material animal foi seco em estufa aerada, sob $70{ }^{\circ} \mathrm{C}$ para remoção de materiais voláteis e composto fenólicos por 12 horas e em seguida triturado em liquidificador. O método de obtenção e purificação do polissacarídeo foi baseado na metodologia proposta por Soares e colaboradores com modificações. ${ }^{22}$

\subsection{Hidrólise do polissacarídeo}

Pesou-se $20 \mathrm{mg}$ do polissacarídeo purificado em tubo de ensaio, que foi posteriormente solubilizado com $1 \mathrm{~mL}$ de água destilada e mantido sob agitação constante por um período de $24 \mathrm{~h}$. Em seguida adicionou-se $1 \mathrm{~mL}$ de $\mathrm{HCl} 4 \mathrm{M}$, sob $100^{\circ} \mathrm{C}$ para início da hidrólise do mesmo. ${ }^{23} \mathrm{~A}$ hidrólise foi acompanhada em espectrofotômetro (Uv-vis) Thermo scientific (modelo Evolution 60S), no comprimento de onda $510 \mathrm{~nm}$ no tempo de 2 , 6 e 8 horas, para verificação de qual tempo apresentava 0 maior teor de monossacarídeos. 0 procedimento foi realizado em triplicata.

\subsection{Infravermelho}

A amostra de polissacarídeo foi analisada em pastilha de $\mathrm{KBr}$, no espectrômetro de infravermelho na região (4000 a $400 \mathrm{~cm}^{-1}$ ) modelo Nexus 470 FT-IR e fabricante Thermo Nicolet.

\subsection{Dosagem de açúcar redutor}

Para a dosagem do açúcar redutor, foi utilizado o método de Nelson Somogyi ${ }^{24}$, o qual consiste na determinação de açúcares redutores solúveis. A quantidade recomendada pela técnica é de $100 \mathrm{mg}$. A curva de calibração foi construída utilizando como padrão a galactose com concentrações variando de 0,$8 ; 1 ; 3 ; 5 ; 7 ; 9$ e $10 \mathrm{mg} / \mathrm{mL}$. As medidas foram feitas em espectrofotômetro semelhante ao descrito no ponto 2.2 deste procedimento.

\subsection{Análise termogravimétrica}

Uma massa de aproximadamente 5,17 mg de material isolado foi aquecida em equipamento SDT-Q 600, fabricante TA, em atmosfera inerte e com taxa de aquecimento de $2,5^{\circ} \mathrm{C} / \mathrm{min}$ com uma variação de $25{ }^{\circ} \mathrm{C}$ a $900{ }^{\circ} \mathrm{C}$.

\subsection{Ressonância magnética nuclear}

O espectro de Ressonância Magnética Nuclear de Hidrogênio (RMN ${ }^{1} \mathrm{H}$ ) unidimensional, foi obtido em espectrômetros Bruker, modelo Avance DRX500, pertencentes ao Centro Nordestino de Aplicação e Uso da Ressonância Magnética Nuclear da Universidade Federal do Ceará (CENAUREMN-UFC). Foi utilizada sonda de detecção inversa de $5 \mathrm{~mm}$, com frequência de $500,13 \mathrm{MHz}\left({ }^{1} \mathrm{H}\right)$, sob um campo magnético de $11,7 \mathrm{~T}$. A amostra foi solubilizada em $0,6 \mathrm{~mL}$ de água deuterada $\left(D_{2} O\right)$, comercializado pela Aldrich. Os deslocamentos químicos (d) foram expressos em partes por milhão ( $\mathrm{ppm}$ ) e 
referenciado pelo pico do hidrogênio pertencente à molécula residual nãodeuterada da água $(d 4,80)$. Os parâmetros de aquisição, respectivamente: larguras espectrais de 24 e 260 ppm, intervalo para relaxação de $1 \mathrm{~s}$ e largura de pulso de $90^{\circ}$ de $9,60 \mathrm{~ms}(0 \mathrm{~dB})$. Foram utilizados 65356 pontos para a aquisição e 32768 para o processamento do espectro.

\section{Resultados e Discussão}

\subsection{Obtenção e purificação do polissacarídeo}

O isolamento de polissacarídeo pode ocorrer por via enzimática ou por extrações com água, seguida de precipitação com etanol. Contudo extrações enzimáticas possuem altos custos, e apesar da seletividade, o rendimento é baixo. Logo, o método adotado foi aquele no qual utiliza-se os solventes de baixo custo, água e etanol e que contemplam os princípios da química verde. ${ }^{14-16,19}$ Os percentuais de polissacarídeo isolado e purificado utilizando este método, foram de $2,9 \%$ e $50 \%$, respectivamente. Foi obtido $30 \mathrm{mg}$ do polissacarídeo purificado, denominado PoliCv, que foi submetido a hidrólise e análises por IV e RMN.

\subsection{Hidrólise e dosagem de açúcar redutor do polissacarídeo}

PoliCv foi submetido a reação de hidrólise por diferentes tempos para otimização do processo. Os tempos de reação utilizados foram 2, 6 e 8 horas. A curva de galactose realizada apresentou índice de correlação $\mathrm{R}^{2}=0.9925$. Para cada um destes tempos concentrações de 1,90; 4,61 e 4,50 mg/mL de material hidrolisado foram identificadas. Desse modo, conclui-se que o melhor tempo de reação é o de 6 horas, tendo em vista que em tempos maiores, a concentração dos monossacarídeos diminui, indicando assim queima ou decomposição das unidades que compõem o polissacarídeo.

\subsection{Infravermelho}

As análises por espectroscopia na região do infravermelho, do polissacarídeo isolado e purificado, não apresentaram mudanças significativas entre eles, e apresentou semelhanças significavas com o polissacarídeo isolado por Chen e colaboradores. ${ }^{25}$ São evidenciadas bandas: $3405 \mathrm{~cm}^{-1}$ pertencentes a deformação axial de grupamento hidroxila; bandas em 2918 e $1415 \mathrm{~cm}^{-1}$ características de deformação axial e angular de hidrogênios ligados a carbonos $\mathrm{sp}^{3}$; sinal intenso relacionado a grupo carbonila em $1646 \mathrm{~cm}^{-1}$; pela alta intensidade da banda $\mathrm{OH}$ em 3405 $\mathrm{cm}^{-1}$ não se observa sinal de ligação $\mathrm{N}-\mathrm{H}$, contudo podemos associá-lo à uma deformação na banda de carbonila com número de onda $1543 \mathrm{~cm}^{-1}$; por fim, percebese, de acordo com a literatura, a presença do grupo sulfato pelo sinal discreto em $1237 \mathrm{~cm}^{-}$ ${ }^{1}$. As bandas apresentam-se destacadas no espectro e vale ressaltar a presença do grupo sulfato, que é o que confere a particularidade aos polissacarídeos de origem marinha, como mostrado na Figura 2. 


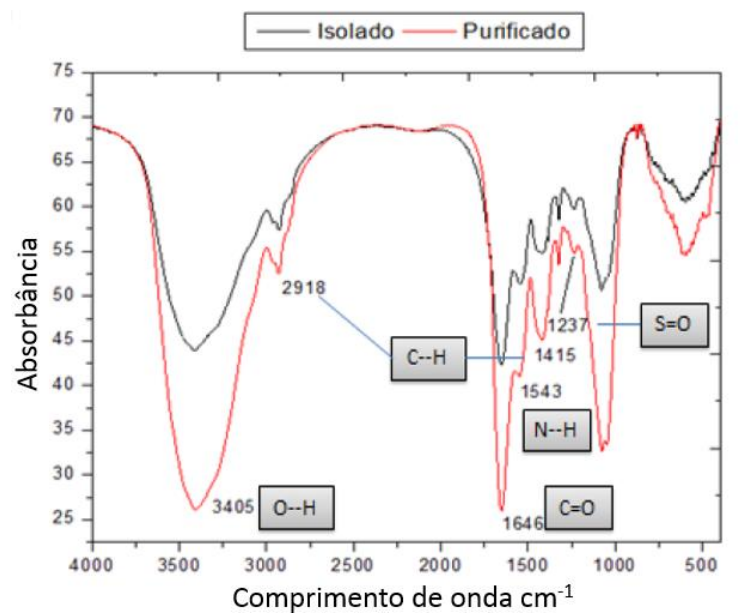

Figura 2: Espectro de infravermelho do polissacarídeo isolado e purificado.

\subsection{Análise termogravimétrica}

A estabilidade térmica é geralmente caracterizada pela temperatura na qual a decomposição do polímero se torna perceptível pela formação de produtos e cinética do processo. Um dos fatores determinantes da estabilidade térmica do polímero é a energia das ligações da cadeia principal. $^{26}$ Dentre as ligações, podemos destacar a ligação $\mathrm{C}-\mathrm{C}$ como umas das mais resistentes a degradação térmica. Os átomos de hidrogênio presentes na molécula aumentam a energia de estabilização da molécula, diminuindo assim a energia envolvida em sua degradação em elevadas temperaturas. ${ }^{27} \mathrm{~A}$ Figura 3 , a seguir, apresenta a curva de TG e DTG do polissacarídeo. $\mathrm{Na}$ curva DTG podemos destacar cinco regiões de degradação, conforme apresentado na Figura 3 abaixo.

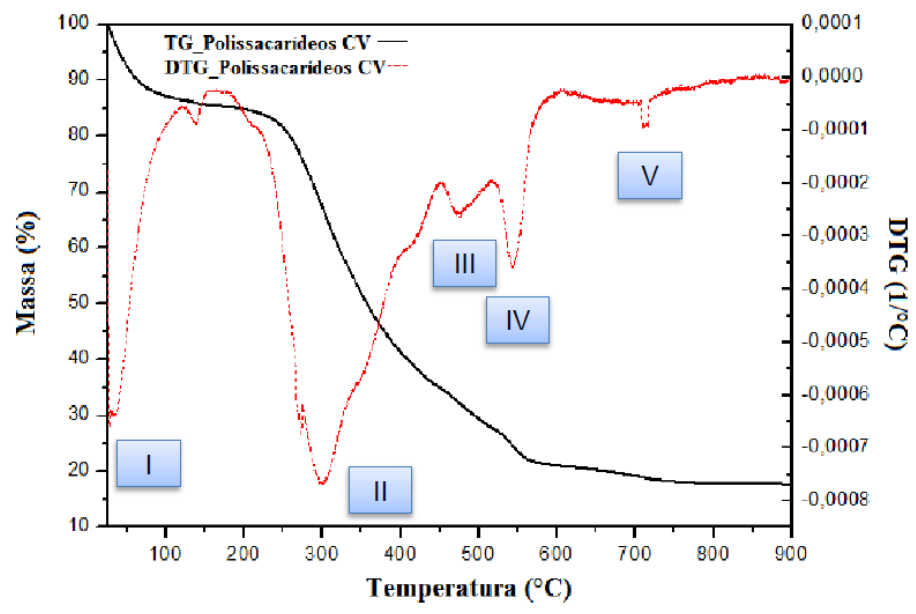

Figura 3: Curva TG e DTG para o polissacarídeo CV.

A região I, é característica de saída de água residual da amostra e corresponde a $15 \%$ da perda de massa total da amostra. As regiões II a IV referem-se à decomposição do material orgânico com liberação de água, dióxido e monóxido de carbono e metano, que corresponde a cerca de $70 \%$ de degradação do material em questão. Por fim, a região $\mathrm{V}$, nos fornece os dados sobre as cinzas, que são pertencentes ao material inorgânico presente 
na amostra ou residual no cadinho, em nosso caso corresponde à $15 \%$ da massa do material, indicando um percentual elevado de sais inorgânicos.

\subsection{Ressonância magnética nuclear}

O espectro de RMN ${ }^{1} \mathrm{H}$ do PoliCv apresentou sinais entre $\delta 3,5$ e 6,0 característicos de hidrogênios ligados à carbonos metino oxigenados, e também sinais entre $\delta 1,5$ e 3,0 que podemos atribuir a hidrogênios ligados a carbono metilênico (diasterotópico). A comparação com dados de RMN de polissacarídeos sulfatados, descritos na literatura, confirma que a estrutura de PoliCv se assemelha a estas estruturas, principalmente com polissacarídeos da classe das carrageninas. Estes polissacarídeos naturais ocorrem com frequência em algas vermelhas, possuem estrutura dimérica, formada por unidades $D$-galactopiranose com padrões glicosídicos $\alpha 1 \rightarrow 3$ e $61 \rightarrow 4$. Os tipos mais comuns de carrageninas são ı (lota), $k$ (kappa) e $\lambda$ (lambda) que se distinguem entre si pelo padrão de substituição do grupo sulfato e teor 3,6 de anidrogalactose, ${ }^{28-30}$ conforme apresentado na Figura 4.

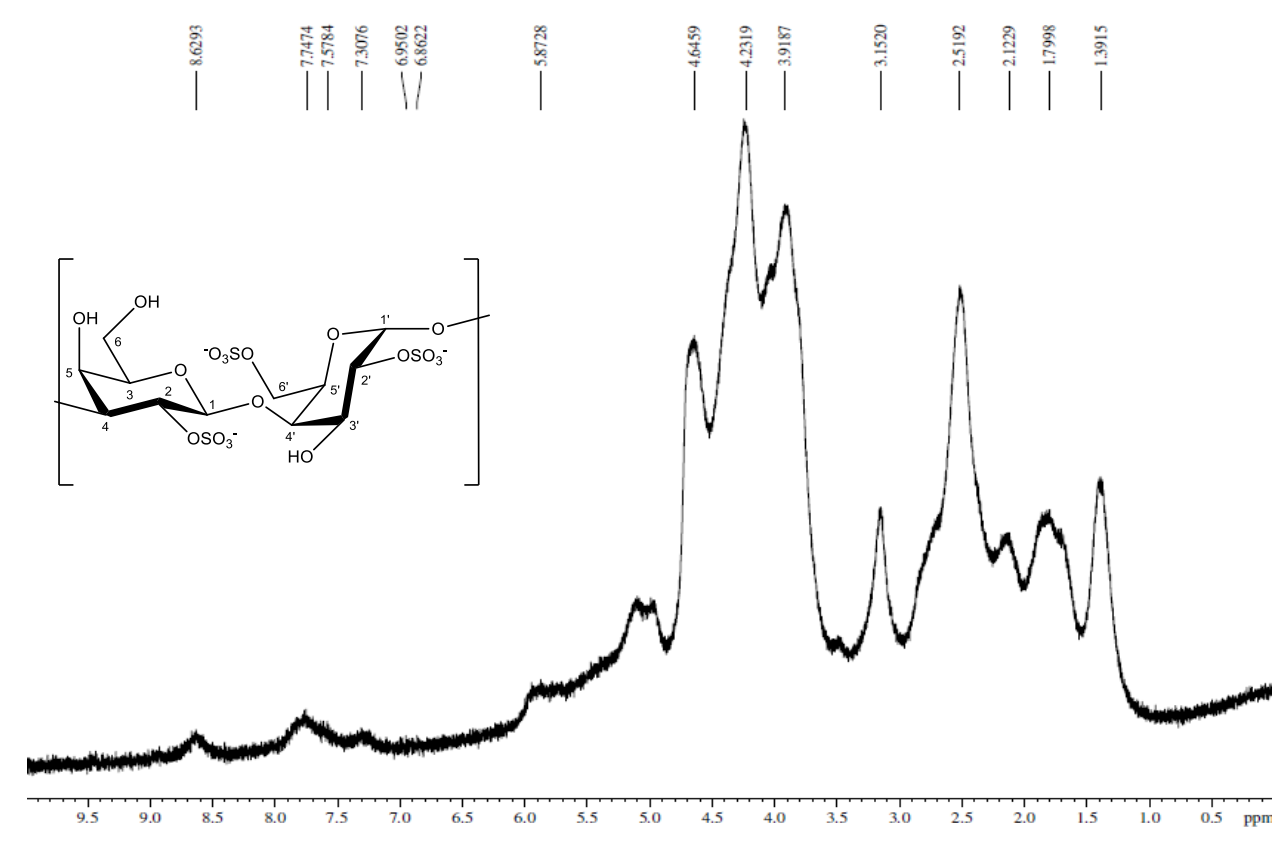

Figura 4: Espectro de $\mathrm{RMN}^{1} \mathrm{H}$ do PoliCv e estrutura química do mero da $D$-galactopiranose para polissacarídeo tipo $\lambda$-carrageninas.

\section{Conclusões}

Com base nos dados apresentados no presente trabalho foi possível comprovar que a espécie $C$. vaginalis se apresenta como fonte de polissacarídeo sulfatado. Não existe diferença significativa entre as amostras isoladas e purificadas, e que devido ao baixo rendimento de cerca de apenas $50 \%$ após a purificação, este pode ser utilizado já na forma isolada, sem tratamento posterior. O melhor tempo de hidrólise foi de 6 horas, apresentando assim concentração de açúcares redutores de $4,60 \mathrm{mg} / \mathrm{mL}$. A análise por infravermelho permitiu identificar grupos amino e sulfato na estrutura do polissacarídeo, compatível com os compostos provenientes de esponjas marinhas. Os dados de RMN confirmaram a presença de polissacarídeo sulfatado na espécie, indicando também um possível mero deste polímero. A análise térmica mostrou que o polissacarídeo apresenta estabilidade até cerca de $200{ }^{\circ} \mathrm{C}$. 
Não existe trabalho na literatura sobre isolamento de polissacarídeos de espécies do gênero Callyspongia, o que torna o trabalho pioneiro e promissor, considerando o potencial biológico das esponjas marinhas e dos polissacarídeos sulfatados.

\section{Agradecimentos}

Os autores agradecem aos órgãos de fomento Capes e CNPq.

\section{Referências Bibliográficas}

${ }^{1}$ Esteves, A. I. S.; Nicolal, M.; Humanes, M.; Goncalves, J. Sulfated polysaccharides in Marine Sponges: Extraction Methods and Anti-HIV Activity. Marine Drugs 2011, 9, 139. [CrossRef]

${ }^{2}$ Ruocco, N.; Constantini, S.; Guariniello, S.; Constantini, M. Polysaccharides from the marine environment with pharmacological, cosmeceutical and nutraceutical potential. Molecules 2016, 21, 551. [CrossRef] [PubMed]

${ }^{3}$ Gochfeld, D. J.; Sayed, K. A. E.; Yousaf, M.; Hu, J. F.; Bartyzel, P.; Dunbar, D. C.; Wilkins, S. P.; Zjawiony, J. K.; Schinazi, R. F.; Wirtz, S. S.; Tharnish, P. M.; Hamman, M. T. Marine natural products as lead anti-HIV agents. Mini Reviews in Medicinal Chemistry 2003, 3, 401. [CrossRef] [PubMed]

${ }^{4}$ Rangel, M.; Falkenberg, M. An overview of the marine natural products in clinical trials and on the market. Journal of Coastal Life Medicine 2015, 3, 421. [CrossRef]

${ }^{5}$ Taylor, M. W.; Radax, R.; Steger, D.; Wagner, $M$. Sponge-associated microorganisms: evolution, ecology and biotechnological potential. Microbiology and Molecular Biology Reviews 2007, 71, 295. [CrossRef] [PubMed]

${ }^{6}$ Archana, R.; Kanchana, G.; Rubalakshmi, G. Free radical scavenging and in vitro anticancer properties breast cancer cell line MCF-7 on Marine sponge Spongia tosta. International
Journal of Current Microbiology and Applied Sciences 2015, 4, 723. [Link]

${ }^{7}$ Kiem, P. V.; Huyen, L. T.; Hang, D. T.; Nguyen, X. N.; Tai, B. H.; Anh, H. L.; Tuan; C., Pham V.; Quang, T. H.; Minh, C. V.; Nguyen, V. D. Sesquiterpene derivatives from marine sponge Smenospongia cerebriformis and their anti-inflammatory activity. Bioorganic \& Medicinal Chemistry Letters 2017, 27, 1525. [CrossRef] [PubMed]

${ }^{8}$ Kobayashi, M.; Higuchi, K.; Murakami, N.; Tajima, H.; Aoki, S. Callystatin A, a potent cytotoxic polyketide from the marine sponge, Callyspongia truncate. Tetrahedron 1997, 38, 2859. [CrossRef]

${ }^{9}$ Umeyama, A.; Matsuoka, N.; Mine, R.; Nakata, A.; Arimoto, E.; Matsui, M.; Shoji, N.; Arihara, S.; Takei, M.; Hashimoto, T. Polyacetylene diols with antiproliferative and driving th1 polarization effects from the marine sponge Callyspongia $s p$. Journal of Natural Medicines 2010, 64, 93. [CrossRef] [PubMed]

${ }^{10}$ Seleghim, M. H. R.; Lira, S. P.; Kossuga, M. H.; Batista, T.; Berlink, R. G. S.; Hadju, E.; Muricy, G.; Rocha, R. M.; Nascimento, G. G. F.; Silva, M.; Pimenta, E. F.; Thiemann, O. H.; Oliva, G.; Cavalcanti, B. C.; Pessoa, C.; Moraes, M. O.; Galetti, F. C. S.; Silva, C. L.; Souza, A. O.; Peixinho, S. Antibiotic, cytotoxic an enzyme inhibitory activity of crude extracts from brazilian marine invertebrates. Revista Brasileira de Farmacologia 2007, 17, 287. [CrossRef]

${ }^{11}$ Capon, R. J.; Ford, J.; Lacey, E.; Gill J. H.; Heiland, K.; Friedel, T. Phoriospongin A and B: two new nematocidal depsipeptides from the australian marine sponges Phoriospongia Sp. and Callyspongia Bilamellata. Journal of Natural Products 2002, 65, 358. [CrossRef] [PubMed]

${ }^{12}$ Lee, Y. E.; Kim, H.; Seo, C.; Park, T.; Lee, K. B.; Yoo, S. Y.; Hong, S. C.; Kim, J. T.; Lee, J. Marine polisaccharide: therapeutic efficacy and biomedical applications. Archives of Pharmacal Research 2017, 40, 1006. [CrossRef] [PubMed] 
${ }^{13}$ Li, S.; Xiong, Q.; Lai, X.; Li, X.; Wan, M.; Zhang, J.; Yan, Y.; Cao, M.; Lu, L.; Guan, J. Molecular modification of polysaccharides and resulting bioactivities. Comprehensive Reviews in Food Science and Food Safety 2016, 15, 237. [CrossRef]

${ }^{14}$ Bucior, I.; Burger, M. M. Carbohydratecarbohydrate interaction as a major force initiating cell-cell recognition. Glycoconjugate Journal 2004, 21, 111. [CrossRef] [PubMed]

${ }^{15}$ Maia, L. F.; Gonzaga, T. A.; Carvalho, R. G.; Leite, C. M.; Lobo-Hadju, G.; Aguiar, J. A. K.; Edwards, H. G. M.; Oliveira, L. F. C. Monitoring of sulfated polysaccharide contente in marine sponges by raman spectroscopy. Vibrational Spectroscopy 2016, 87, 149. [CrossRef]

${ }^{16}$ Witvrouw, M.; Declercq, E. Sulfated polysaccharides extracted from sea algae as potential antiviral drugs. General Pharmacology 1997, 29, 497. [CrossRef] [PubMed]

${ }^{17}$ Cimino, P.; Bifulco, G.; Casapullo, A.; Bruno, I.; Gomez-Paloma, L.; Riccio, R. Isolation and NMR characterization of rosacelose, a novel sulfated polysaccharide from the sponge Mixylla rosacea. Carbohydrate Research 2001, 334, 39. [CrossRef] [PubMed]

${ }^{18}$ Cunha, P. L. R.; Paula, R. C. M.; Feitosa, J. P. A. Possacarídeos da biodiversidade brasileira: uma oportunidade de transformar conhecimento em valor econômico. Química Nova 2009, 32, 649. [CrossRef]

${ }^{19}$ Zierer, M. S.; Mourão, P. A. S. A wide diversity of sulfated polysaccharides are synthesized by different species of marine sponges. Carbohydrate Research 2000, 328, 209. [CrossRef] [PubMed]

${ }^{20}$ Anastas, P. T.; Warner, J. C. Green chemistry: Theory and practice. Oxford University press: New York, 1998.

${ }^{21}$ Erythropel, H. C.; Zimmerman, J. B.; Winter, T. M.; Petitjean, L.; Melnikov, F.; Lam, C. H.; Lounsbury, A. W.; Mellor, K.E.; Jankovic, N. Z.; Tu, Q. The Green ChemisTREE: 20 years after taking root with the 12 Principles. Green Chemistry 2018, 20, 1929. [CrossRef]

${ }^{22}$ Soares, C. M.; Malagoli, B. G.; Menezes, G. B.; Pinho, V.; Souza, D. G.; Teixeira, M. M.;
Braga, F. Antiadhesive activity of polysaccharide-rich fractions from Lithothamnion muelleri. Verlag der Zeitschrift fur Naturforschung 2012, 67, 391. [PubMed]

${ }^{23}$ Yang, B.; Yu, G.; Zhao, X.; Jiao, G.; Ren, S.; Chai, W. Mechanism of mild acid hydrolysis of galactan polysaccharides with highly ordered disaccharide repeats leading to a complete series of exclusively odd-numbered oligosaccharides. The FEBS Journal 2009, 276, 2125. [CrossRef] [PubMed]

${ }^{24}$ Maldoanade, I. R.; Carvalho, P. G. B.; Ferreira, N. A.; Moulin, B. S. F. Protocolo pra determinação de açúcares redutores pelo método de Somogyi-Nelson. Comunicado técnico, Embrapa ISSN 1414.9850 [Link]

${ }^{25}$ Chen, D.; Yao, W.; Zhang, X.; Han, X.; Qu, X.; Ka, W.; Sun, D.; Wu, X. Effects of gekko sulfated polysaccharide-protein complex on human hepatoma smmc-7721 cells: inhibition of proliferation and migration. Journal of Ethnopharmacology 2010, 127, 702. [CrossRef] [PubMed]

${ }^{26}$ Lim, L. Y.; Wan, L. S. C. Heat treatment of chitosan films. Drug Development and Industrial Pharmacy 1995, 21, 839. [CrossRef]

27 Damian, C.; Beirão, L. H.; Francisco, A.; Santo, M. L. P. E.; Teixeira, E. Quitosana: um amino polissacarídeo com características funcionais. Alimentos e Nutrição 2005, 16, 195.

${ }^{28}$ Webber, V.; Carvalho, S. M.; Ogliari, P. J.; Hayashi, L.; Barreto, P. L. M. Optimization of the extraction of carrageenan from Kappaphycus alvarezii using response surface methodology. Ciência e Tecnologia de alimentos 2012, 32, 812. [CrossRef]

${ }^{29}$ Korva, H.; Karkkainen, J.; Lappalainen, K.; Lajunen, M. Spectroscopic study of natural and synthectic polyssaccharide sulfate structures. Starch 2016, 68, 854. [CrossRef]

${ }^{30}$ Gonçalves, A. G.; Ducatti, D. R. B.; Paranha, R. G.; Eugenia, M.; Duarte, R.; Noseda, M. D. Positional isomers of sulfated oligosaccharides obtained from agarans and carrageenans: preparation and capillary electrophoresis separation. Carbohydrate Research 2005, 340, 2123. [CrossRef] [Pubmed] 